\title{
Design Practice of Tourist Road Based on Rural Revitalization -Taking Jinan S103 Tourist Highway as an Example
}

\author{
Wang Wei-gang, Xu Ming, Zhou Jian, Wang Qi \\ China Academy of Transportation Science, Beijing, 100029
}

\begin{abstract}
Combining with the design practice of Jinan S103 tourist road, analyzing the design principles of tourist road and the difficulty of project implementation, the author proposes to build five major systems of tourist road to promote tourist road construction.
\end{abstract}

\section{INTRODUCTION}

The report of the 19th National Congress of the Communist Party of China put forward the strategy of vigorously implementing the rural revitalization and development, which also implemented to solve the fundamental problem related to the national economy and the people's livelihood. The countryside is usual a regional complex of high-quality natural resources and environment, simple folk customs and social structure, relatively underdeveloped and lack of economic sources. It also has multiple functions such as production, life, ecology, and culture. Urbanization provides mutual promotion and coexistence, and together constitute the main space for urban and rural dualization activities. The beautiful natural scenery, excellent ecological environment, and high-quality agricultural resources in rural areas promote the emergence of rural tourism, and provide tourists with new types of tourism industry products, making rural tourism an important tool for promoting rural development. In order to develop rural tourism, the transportation infrastructure in rural areas must be constructed so that the vast number of tourists can quickly, conveniently, safely and comfortably reach rural areas for tourism, and promote the merging of transportation and tourism. Besides, the practice can improve the construction of transportation infrastructure, provide tourists with a new experience of "beauty in roads, beauty in service", build a "fast-forward and slow-travel" tourism transportation network, and implement the virtuous circle of rural revitalization and integrated development of transportation and tourism.

This article combines the design practice of the tourist road from the south of Shi Zhong Hong Fu Road in Jinan S103 Jizao line to Jinan Tai'anjie (hereinafter referred to as this project), and discusses the design of tourist roads under the background of rural revitalization that implement the development concept of transportation and tourism integration.

\section{PROJECT IMPLEMENTATION BACKGROUND}

\subsection{Locational Conditions of the Project}

The project is located in the southern mountain area of Jinan City, about 10 kilometers away from the Jinan urban area. S103 tourist highway is not only the main passage from Jinan urban area to Zhonggong Town and Liubu Town in the southern mountain area, but also an extremely important economic development axis in the southern mountainous area. After the implementation of the south control strategy for the development of Jinan City, the quality of the ecological environment in the southern mountainous area has been further improved, which is very suitable for rural tourism. The southern mountain area is much known as the water source and backyard garden of Jinan, the provincial capital spring city.

\subsection{Tourism and Product Resources of the Project}

The southern mountainous area is located nearby Mount Tai, with a unique geographical location, surrounded by beautiful mountains, river and ravines, with a forest coverage rate of $57 \%$. There are natural scenic spots such as Jiurushan Waterfalls and Hongye Valley in the area, as well as many leisure and entertainment venues such as the Golden Elephant Mountain Park and the Nine-top Tower Chinese Folklore Amusement Park. At the same time, the walnut, peach, pear, chicken and other agricultural specialty products in the southern mountainous area are also of various kinds and high-quality, which is very suitable for the environmental requirements and purchase needs of urban residents nowadays.

\footnotetext{
* Corresponding author: 308517829@qq.com
} 


\subsection{The Project does not Meet the Tourism Needs}

Due to the abundant tourism resources and superior locational conditions in the southern mountainous area where the project is located, tourism travel has increased, especially during the holidays. S103 line traffic congestion is serious, which can no longer meet the needs of regional tourism development and residents travel nowadays. This situation severely restricts the development of local tourism and economy, and is not conducive to the implementation of rural revitalizationor the completion of poverty alleviation tasks.

\subsection{Obstacles to the Project Development of Rural Tourism Economy}

The distribution of tourism resources in the project area is relatively scattered and lacks connection. Through tourism roads as carriers, tourism resources can be integrated and connected. the cultural depth of rural tourism products required for the development of rural tourism economy is insufficient. Promote and promote the role to increase popularity, and transfer to the later optimization and in-depth in the development process of the tourism economy. When developing the rural tourism economy, how to coordinate with the ecological environment, solve the fragile ecological environment, meet the requirements of the province's southern control strategic planning, and break the obstacles such as the single development mode of the rural tourism industry and the weak linkage is worthy of discussion and consideration.

\section{TOURISM ROAD DESIGN PRACTICE}

Analysing the implementation background of the comprehensive project, Based on the people's growing demand for a better travel environment and the need for the integrated development of transportation and tourism nowdays, with the consideration of avoiding the destruction of the natural environment along the route by road construction, and at the same time to promote the development of local tourism, the design create a Shandong characteristic typical demonstration tourist road project During the design practice, designers establishe the design principles of transportation and travel integration, analyze the difficulties of tourist road construction, improve the tourist road system, and discusse the road + ecological tourism construction model.

\subsection{Principles of Tourist Road Construction}

In the long-term highway construction process, we have accumulated abundant experience and formed more systematic and perfect highway construction principles and concepts. As a new extension of the type of highway construction, the tourist road fully draws on the road development concept of "innovation, coordination, green, openness and sharing". We should combine the requirements of tourist road construction and the specific environmental characteristics of the project to clarify the design principles ,in order to facilitate the tourist road construction proceeding well.

(1) The principle of safety first

The primary goal of road construction is to ensure the safety of road users. During construction, safety should be put first, and all effective methods and measures should be taken to ensure the safety of road facilities, the safety of running vehicles and the safety of pedestrians, so that users can be well Land use and experience tourist roads.

(2) Principles of serving the public

The construction of tourist roads can drive the economic development of the areas along the route, show the local customs and customs along the route, promote social progress and development, and make positive contributions to the construction of the harmonious society. In the concrete implementation of the project, based on the principle of serving the public, considering the needs of different groups of people, it is necessary to minimize interference with residential villages and schools, protect valuable historical sites and other cultural landscapes, improve services, and effectively improve road quality.

(3) The principle of respecting regional characteristics

The area where the project is located has its own geographic location, topographic features, Plant varieties, climatic characteristics, and social environmental characteristics. When referring to the successful construction experience of domestic and foreign tourist roads, the local characteristics of the project should be taken into consideration to build its own landscape and reflects the characteristics of the region.

(4) The principle of overall natural coordination

Tourism road is a linear project, and its corridor landscape construction should consider the horizontal and vertical lines of the highway itself,. Highway structures, facilities along the route, the surrounding terrain, landforms, ecological features, and other natural and human landscapes should be considered as a whole. This artificial product is coordinated with the natural system along the route, so that the tourist road can improve the original landscape environment under the premise of fast-forward roaming.

\subsection{Difficulties in Tourist Road Construction}

The tourist road emphasizes "slow travel". How to do everything possible to keep people stay or slow down requires that the tourist road must build its own characteristic products, which is the key point in the construction of the tourist road. There are abundant resources of moutains and rivers along the project, which connects nine tourist branches and directly connects thirty-five tourist attractions. There are beautiful scenery in four seasons and rich agricultural specialty resources. It is close to the provincial capital Jinan and has huge tourism market potential. The project's creation of featured products expands from the excavation of 
historical and cultural accumulations along the road, the development of excellent natural scenery resources along the route, and the creation of Internet celebrity spots that can attract tourists to come and play repeatedly and realize the internal circulation of tourism. Different projects have different conditions of their own resources, which requires that the construction of tourist roads needs to be planned scientifically and specifically based on the specific actual resources of the project. To form a complete tourist road construction idea, we should creat the changed road scenery, grasp the positioning, innovation and expansion of tourist road construction.

\subsection{Five Systems of Tourist Roads}

Analyze the project's own conditions and construction difficulties, combine the needs of Shandong Province to build a strong transportation country, establish the project to become an innovative road for strong transportation country, to become a demonstration road for high-quality development of ordinary national and provincial roads, to combine tourism and transportation with other industries, to contribute ecological roads that closely integrate roads and scenery, and to build a win-win road for interactive development between Jinan and Tai'an. These five goals are integrated into the overall goal of creating a fast-arriving fast-track road system, a slow-moving road system for leisure experience while walking, The five systems of ecologically-first environmental protection landscape system, people-oriented tourism service system, and smart information system supported by big data create complete tourism road products.

(1) Fast road system

This project belongs to the reconstruction and expansion of the old road. It is based on the topography, geology, hydrology and other natural conditions along the line, combined with the development plan of the main towns along the line, the road network layout, and the coordination of the surrounding environment, etc. According to the overall direction of the route, and on the premise of meeting technical standards, the project make use of old roads as much as possible. The whole line either widen on both sides or widenon one side, rationally lay out subgrade sections, level intersections, etc., optimize the road driving environment, create a fast-moving road system that can pass quickly, and meet the "fast-forward" of tourist roads need.

(2) Slow road system

In order to increase the travel experience, allow tourists to achieve the goal of "slow travel" and meet the needs of green travel, the project combines tourism resources around the fast-moving road system and flexibly arrange bicycle and walking paths to facilitate the use of tourists and serve travelers along the road, achieving a win-win situation between road construction and economic development along the route. The bicycle lanes are divided according to the characteristics of the whole road landscape, combined with the local history and culture, and pay attention to the color, pattern, and cultural influence of the slow lanes to increase the fun of the road surface, seal the literati poems, etc., in order to strengthen the promotion of local humanities and history.

(3) Environmental protection landscape system

Road construction is bound to bring certain damage and impact to the environment along the route, and at the same time, as a tourist road, it also has high requirements on the landscape. Ecological restoration and environmental protection measures and landscape construction in the later period are very necessary. The natural and human environment along the project has changed significantly. According to the different characteristics of the road, the design along the line will be built into three landscapes: "Discovering the peace from the city, watching the mountain view by the river, and climbing the mountain to enjoy the scenery ". The three parts of the road display through the plant landscape, hydrophilic landscape, and mountain natural landscape, provides visitors with spring flowers and autumn leaves, summer coolness, water play, outdoor exploration and other experiences and fun, enriching the experience of tourist road products.

(4) Tourism service system

Tourism emphasizes the supremacy of service. Only with good service can we provide tourists with a better experience. While accumulating word-of-mouth popularity and expanding tourism brand awareness, can tourists have the possibility of repeated experiences. The tourist road service facilities need to meet the needs of different groups of people such as self-driving, cycling, hiking, etc. Facilities are arranged along the tourist road, connected with the fast or (and) slow-moving system, and are specially provided for tourist road users. Service content such as tourism information, rest environment, dining venues, accommodation services, emergency facilities, and road service facilities with additional functions such as viewing, popular science, sales, and cultural exhibitions.

The land for tourism service facilities is closely integrated with engineering construction, minimize the newly added land, make full use of the land after the optimization of the highway alignment, classify the service facilities, and distinguish the different types of scales and functions, which not only meets the overall needs, but also meets the needs of tourism in different locations.

(5) Smart Information System

Tourists need to obtain more information in order to accurately grasp tourist information and make arrangements for themselves. The tourist road is required to provide thavelers with better services. The design including implementing Wifi coverage, building a smart travel service platform, adding a common sense device, and a big data collection system, not only provide more convenient transportation, accommodation, entertainment, catering and consumption mode for tourists but also provide managers with more intuitive and effective travel data.

\section{CONCLUSION}

This project is a major practice in the construction of Shandong tourist roads, and strives to create a 
comprehensive fast and slow transportation and tourism corridor in the province with perfect services. It will form a "fast forward and slow travel" tourism pattern, which is a "road + ecological tourism" construction model in Jinan City. The new attempt is a new way to realize the transformation of highway transportation infrastructure from purely satisfying travel functions to multiple functions such as transportation, ecology, cultural communication, tourism, and consumption. It is also a demonstration road for ordinary national and provincial trunk lines of the upgraded version of "smooth, safe, comfortable and beautiful".

The tourist road can well display the regional economic development and social image, and is conducive to promoting and driving regional development. But it can't solve all the problems. It is necessary to do a good job in regional overall planning, special tourism planning, and specific tourism products and construction. What kind of culture can really attract people, transform high-quality tourism and natural environmental resources into attracting more people, build a tourist road brand, and lead the development of tourist road construction.

\section{REFERENCES}

1. Liu Jinhong. Research on the Sustainable Development of Rural Tourism in the Southern Mountain Area of Jinan. Urban Tourism Planning. 2019.7

2. Liu Yafei. Research on key technical standards for expressway service facilities. Chang'an University. Master's degree thesis. 2012.6

3. Zhu Qiang, Yu Kongjian, Li Dihua. Ecological corridor width in landscape planning. Acta Ecologica Sinica. 2005

4. Gu Xiaofeng, Kong Yaping, Chen Bing. Research and Practice on Planning and Design of Tourist Highway Service Facilities. China Engineering Consulti 